\title{
Two-Year US Pharmacovigilance Report on Brodalumab
}

\author{
Mark Lebwohl · Craig Leonardi · Jashin J. Wu • April Armstrong · \\ Nicole Rawnsley · Mohammed Merchant · Binu Alexander • \\ Abby Jacobson
}

Received: October 27, 2020 / Accepted: November 26, 2020 / Published online: December 18, 2020

(C) The Author(s) 2020

\section{ABSTRACT}

Introduction: Brodalumab is a human interleukin-17 receptor A antagonist indicated for the treatment of moderate-to-severe plaque psoriasis in adult patients who are candidates for systemic therapy or phototherapy and have failed to respond or have lost response to other systemic therapies. In the United States, brodalumab carries a boxed warning about suicidal ideation and behavior; however, no causal association was established between brodalumab and suicides reported during pivotal trials. We have previously

\author{
M. Lebwohl $(\bowtie)$ \\ Icahn School of Medicine at Mount Sinai, New York, \\ NY, USA \\ e-mail: lebwohl@aol.com \\ C. Leonardi \\ Saint Louis University School of Medicine, St. Louis, \\ MO, USA \\ J. J. Wu \\ Dermatology Research and Education Foundation, \\ Irvine, CA, USA \\ A. Armstrong \\ University of Southern California, Los Angeles, CA, \\ USA \\ N. Rawnsley · A. Jacobson \\ Ortho Dermatologics (a division of Bausch Health \\ US, LLC), Bridgewater, NJ, USA \\ M. Merchant · B. Alexander \\ Bausch Health, Bridgewater, NJ, USA
}

reported results from an analysis of 1-year pharmacovigilance data in patients in the United States who took brodalumab, in which the most commonly reported adverse event was psoriasis flare. There were no completed suicides, suicide attempts, or serious fungal infections. Here, we provide a 2-year US pharmacovigilance report.

Methods: This analysis summarizes pharmacovigilance data reported to Ortho Dermatologics by US patients and healthcare providers from August 15, 2017, through August 14, 2019. The most common adverse events listed in the brodalumab package insert (incidence $\geq 1 \%$; arthralgia, headache, fatigue, diarrhea, oropharyngeal pain, nausea, myalgia, injection-site reactions, influenza, neutropenia, and tinea infections) and adverse events of special interest are reported.

Results: Data were collected from 2677 patients in the United States who took brodalumab, with an estimated exposure of 1656 patient-years. Arthralgia was the most commonly reported adverse event (73 events; 0.04 events per patient-year). No suicide attempts or completed suicides were reported; there were 25 reports of depression. There were 46 serious infections and no serious fungal infections. One event of Crohn's disease was reported, which led to discontinuation. There were 13 malignancies, with none deemed related to brodalumab.

Conclusions: This pharmacovigilance report supports the safety profile of brodalumab previously reported from long-term analyses of clinical trials and 1-year pharmacovigilance data. 
Keywords: Adverse events; Brodalumab; Pharmacovigilance; Psoriasis; Real-world data; Safety; Suicidal ideation and behavior

\section{Key Summary Points}

Why carry out this study?

Adult patients with moderate-to-severe plaque psoriasis who have failed to respond to systemic therapies or phototherapies may respond to brodalumab, a treatment option that has been shown to be efficacious in this patient population.

Because psoriasis is a chronic disease that requires long-term treatment, it is important to assess long-term data regarding common adverse events and events of special interest in a real-world setting, including suicidal ideation and behavior (which occurred in patients treated with brodalumab during pivotal trials, although no causal association was established).

Focusing on the most common adverse events listed in the brodalumab package insert and adverse events of special interest, this analysis summarizes brodalumab pharmacovigilance data reported to Ortho Dermatologics by US patients and healthcare providers from August 15, 2017, through August 14, 2019 , to provide a clear safety profile of brodalumab.

\section{What was learned from the study?}

The most commonly reported adverse event was arthralgia, there were 46 serious infections and no serious fungal infections, and there were 13 reports of malignancies that occurred in 10 patients (none of which were deemed related to brodalumab); notably, there were no reports of suicide attempts or completed suicides.
This 2-year US pharmacovigilance report supports the safety profile of brodalumab previously reported in long-term analyses of clinical trials and 1-year pharmacovigilance data and may assist healthcare providers when choosing the best treatment option for patients with psoriasis.

\section{DIGITAL FEATURES}

This article is published with digital features to facilitate understanding of the article. You can access the digital features on the article's associated Figshare page. To view digital features for this article go to https://doi.org/10.6084/m9. figshare.13288742.

\section{INTRODUCTION}

Brodalumab is a human interleukin (IL)-17 receptor A antagonist indicated for treatment of moderate-to-severe plaque psoriasis in adult patients who are candidates for systemic therapy or phototherapy and have failed to respond or have lost response to other systemic therapies. The safety profile has been described in one phase 2 and three phase 3 trials (AMAGINE$1 /-2 /-3$ ) [1-4]. Although US prescribing information for brodalumab carries a boxed warning about suicides, causal association between brodalumab and suicides reported during pivotal trials has not been established [1].

In long-term analyses of the AMAGINE-1 and AMAGINE-2 studies, arthralgia, headache, diarrhea, oropharyngeal pain, and Candida infections were among the most frequent adverse events (AEs) with brodalumab treatment over 120 weeks $[5,6]$. We previously reported 1-year US pharmacovigilance AE data of brodalumab [7]. In the 1-year analysis, there were no completed suicides, suicide attempts, or serious fungal infections. The most commonly reported AEs were psoriasis flare, drug ineffectiveness, arthralgia, depression, diarrhea, and pain. In the current analysis, we review 
2-year brodalumab pharmacovigilance data in the United States.

\section{METHODS}

We summarize AEs reported to Ortho Dermatologics between August 15, 2017, and August 14, 2019, which were collected from 2677 US patients who took brodalumab. Adverse events were reported through pharmacovigilance channels for patients and healthcare providers (HCPs). This analysis focuses on the real-world occurrence of the most common AEs listed in the brodalumab package insert (incidence $\geq 1 \%$ ) including arthralgia, headache, fatigue, diarrhea, oropharyngeal pain, nausea, myalgia, injection-site reactions, influenza, neutropenia, and tinea infections [1]. Additionally, AEs of special interest are summarized with descriptive statistics and as exposure-adjusted rates per patient-year (PY).

Brodalumab exposure was conservatively estimated as $1656 \mathrm{PY}$ in the United States by calculating the first shipment date to last dosing date plus 55 days, equating to 5 half-lives of brodalumab. This calculation most likely represents an underestimation as these data were derived from a subset of 13 contracted pharmacies and noncontracted pharmacies that filled brodalumab prescriptions using a copay reimbursement card. Therefore, exposure rates do not include individuals who were treated at noncontracted pharmacies that did not use a copay reimbursement card. Additionally, pharmacovigilance reports did not typically describe medications from which patients were transitioning, provide detailed medical histories of patients, or specify duration of time from last therapy to brodalumab initiation.

The postmarketing data used in this analysis were noninterventional and were not collected as part of a clinical study; therefore, ethics approval was not required. This article is based on previously conducted studies and does not contain any new studies with human participants or animals performed by any of the authors.

\section{RESULTS}

\section{Real-World Occurrence of AEs Listed in Prescribing Information}

In this 2-year analysis, $15.1 \%$ of AEs were reported by HCPs, whereas $84.9 \%$ were reported by patients or non-HCPs. Common AEs from the brodalumab package insert that were assessed are listed in Table 1. Arthralgia was the most common $\mathrm{AE}$ listed in the package insert for patients receiving brodalumab for 12 weeks in clinical trials $(n=71 ; 4.7 \%)$ [1]. It was also among the most frequently reported AEs in long-term analyses of AMAGINE- 1 ( $n=100 ; 7.5$ events per 100 PY) [5] and AMAGINE-2 ( $n=295 ; 9.1$ events per $100 \mathrm{PY}$ ) [6] and in the 1-year pharmacovigilance analysis $(n=16 ; 0.07$ events per PY) [7]. In this 2-year analysis, there were 73 reports of arthralgia (0.04 events per PY; Table 1). At the time of this analysis, it was reported that of these 73 patients, 30 continued and 20 discontinued brodalumab treatment; 23 actions were unknown. Arthralgia was most frequently reported for patients aged 40 to 50 years $(n=33)$, followed by patients aged 60 to 90 years $(n=19)$. Of 73 patients with arthralgia, 9 had concomitant myalgia and 19 had associated comorbid conditions (psoriatic arthritis, prior joint pain, possible arthritis, or other autoimmune conditions).

Other common AEs listed in the brodalumab package insert include headache, fatigue, diarrhea, oropharyngeal pain, nausea, myalgia, injection-site reactions, influenza, neutropenia, and tinea infections. In this analysis, there were no reports of neutropenia or tinea infections (Table 1). Of 25 patients experiencing injectionsite reactions, 15 maintained brodalumab treatment and 1 discontinued. All injection-site reactions were reported by patients or nonHCPs. Most patients experiencing headache $(16 / 28)$ and fatigue $(17 / 23)$ were female, and there were 5 and 4 known cases of patients discontinuing brodalumab owing to headache and fatigue, respectively. Four patients experiencing headache also reported fatigue. Most patients who experienced fatigue were aged 40-70 years. Most patients who experienced 
Table 1 US pharmacovigilance monitoring of brodalumab through 2 years (August 15, 2017-August 14, 2019)

\begin{tabular}{|c|c|c|c|c|c|}
\hline $\mathbf{A E}$ & $\begin{array}{l}\text { Patients } \\
\text { experiencing } \\
\text { event, } n(r)^{\mathrm{a}}\end{array}$ & $\begin{array}{l}\text { Event related to } \\
\text { brodalumab, } n^{\text {b }}\end{array}$ & $\begin{array}{l}\text { Brodalumab } \\
\text { discontinued, } \\
n(\%)^{c}\end{array}$ & $\begin{array}{l}\text { Brodalumab } \\
\text { maintained, } \\
n(\%)^{c}\end{array}$ & $\begin{array}{l}\text { Action unknown } \\
\text { or NA, } n(\%)^{c}\end{array}$ \\
\hline Arthralgia & $73(0.04)$ & 1 & $20(27)$ & $30(41)$ & $23(32)$ \\
\hline Fatigue & $23(0.01)$ & 1 & $4(17)$ & $14(61)$ & $5(22)$ \\
\hline Diarrhea & $24(0.01)$ & 0 & $4(17)$ & $14(58)$ & $6(25)$ \\
\hline $\begin{array}{l}\text { Injection-site } \\
\text { reaction }\end{array}$ & $25(0.02)$ & 0 & $1(4)$ & $15(60)$ & $9(36)$ \\
\hline Headache & $28(0.02)$ & 0 & $5(18)$ & $18(64)^{\mathrm{d}}$ & $5(18)$ \\
\hline Myalgia & $21(0.01)$ & 0 & $7(33)$ & $10(48)$ & $4(19)$ \\
\hline Nausea & $19(0.01)$ & 0 & $3(16)$ & $13(68)$ & $3(16)$ \\
\hline $\begin{array}{l}\text { Oropharyngeal } \\
\text { pain }\end{array}$ & $14(0.01)$ & 0 & $1(7)$ & $8(57)^{\mathrm{e}}$ & $5(36)$ \\
\hline Influenza & $13(0.01)$ & 0 & $4(31)$ & $5(38)$ & $4(31)$ \\
\hline Neutropenia & 0 & 0 & - & - & - \\
\hline Tinea infection & 0 & 0 & - & - & - \\
\hline \multicolumn{6}{|c|}{$\begin{array}{l}A E \text { adverse event; } N A \text { not applicable; } r \text { exposure-adjusted rate per patient-year } \\
\text { a Number of patients experiencing AE, not total number of AEs } \\
\text { b Relatedness to brodalumab was based on company-determined causality } \\
\text { c Treatment action taken upon AE occurrence. Percentage is } n \text { divided by total number of patients experiencing event } \\
\text { d One patient had the drug placed on hold }\end{array}$} \\
\hline
\end{tabular}

fatigue also had or potentially had an underlying comorbid condition [anemia, arthritis, or hypothyroidism (16/23)], and 7 were also receiving concomitant medications associated with fatigue (including central nervous system depressants and antihistamines). Of 13 influenza cases, 8 occurred during months associated with flu season (October to May [8]). Most influenza accounts (12/13) were reported by patients or non-HCPs. For most AEs, reporters often provided no medical history or medication list, limiting a full understanding of potential underlying conditions.

\section{Adverse Events of Special Interest}

Adverse events of special interest included suicide, depression, serious infections, fungal infections, Crohn's disease, and malignancy (Fig. 1). There were no reports of suicide attempts or completed suicides. There were 25 reports of depression ( 0.015 events per PY); of these patients, 6 had a history of depression, 2 reported no history of depression, and 17 did not provide any details as to their mental health history. There were 46 reports of serious infections (0.028 events per PY), with 36 possibly related and 6 unlikely related to brodalumab (4 were nonassessable). Of patients with serious infections, 18 continued and 15 discontinued brodalumab; 13 actions were unknown. There were no reported serious fungal infections; 4 AEs of nonserious fungal infections led to brodalumab discontinuation (2 oral fungal infections [1 yeast infection of mouth], 1 fungal infection of fingernail, and 1 unclassified fungal 


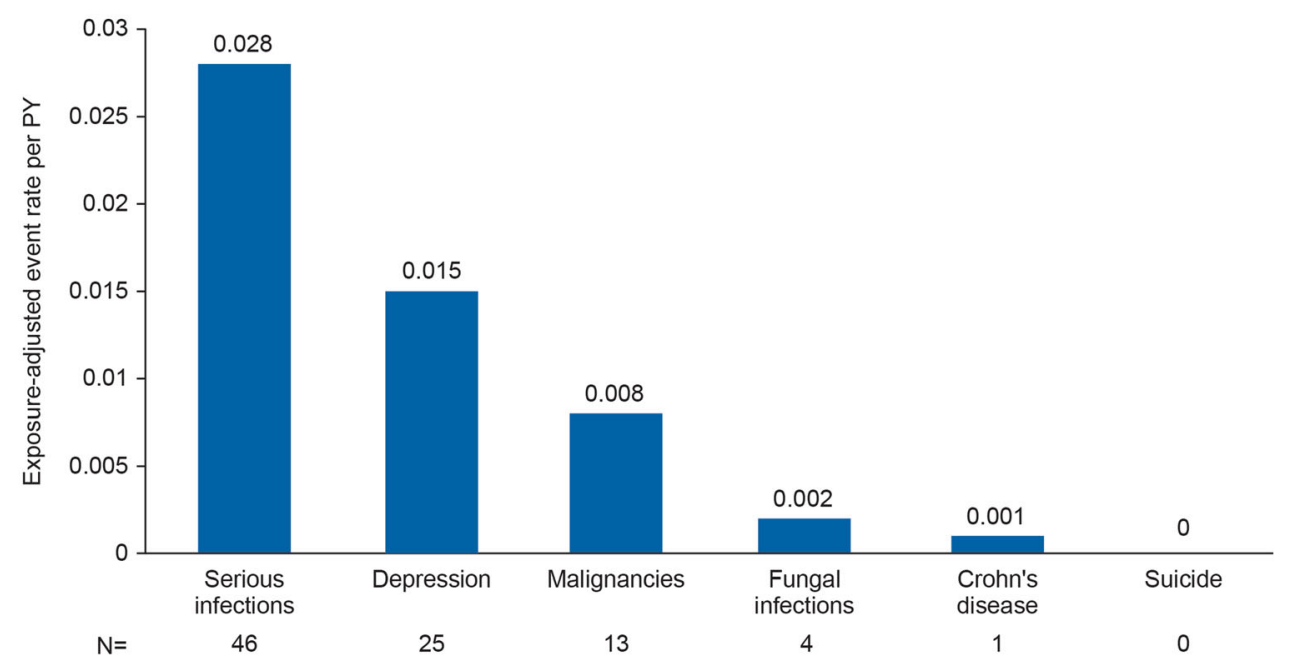

Fig. 1 Exposure-adjusted AEs of special interest per PY. The exposure-adjusted event rate per PY is the number of events per 1656 PY of exposure. $A E$ adverse event; $P Y$ patient-year

infection). There were 2 oral candidiasis reports, for which 1 patient continued treatment and 1 action was unknown. One patient-reported $\mathrm{AE}$ of Crohn's disease (0.001 events per PY), leading to discontinuation, occurred in a patient with a 2-year history of unexplained anemia.

Malignancy rates reported in a combined 1-year analysis of AMAGINE-1/-2/-3 and in a 1 -year US pharmacovigilance analysis were 0.9 events per $100 \mathrm{PY}$ and 0.01 events per PY, respectively $[7,9]$. In this 2-year analysis, there were 13 malignancy reports that occurred in 10 patients ( 0.008 events per PY), which included 3 cases previously reported in the 1-year analysis [7]. Reported malignancies included ovarian, hepatic, lung, prostate, renal, gallbladder, skin cancer, squamous cell carcinoma of the skin after plaque improvement, keratoacanthomatype squamous cell carcinoma, carcinoma in leg, and 3 neoplasms (tumor in neck, benign growth on leg, and malignant tumor removed from arm). Ovarian, hepatic, and lung malignancies were reported in the previous 1-year pharmacovigilance analysis [7]. No malignancies were deemed related to brodalumab. Of the 13 malignancy cases, 7 and 5 were associated with continued and discontinued treatment, respectively (action was unknown for 1 case).

\section{DISCUSSION}

This US pharmacovigilance report on brodalumab summarizes the most common AEs from the brodalumab package insert and AEs of special interest reported from August 15, 2017, through August 14, 2019. There were no reports of suicide attempts or completed suicides. There were 46 serious infections and no serious fungal infections.

Previously, a 1-year report of pharmacovigilance data of brodalumab found that the most commonly reported AE was psoriasis flare [7]. Similar to this 2-year update, there were no reports of suicide attempts or completed suicides. This 2-year analysis investigated the most common AEs from the brodalumab package insert because many of the "most common" AEs listed in the 1-year report did not include detailed information because of the unsolicited manner of pharmacovigilance reporting.

In this 2-year update, the most commonly reported $\mathrm{AE}$ listed in the brodalumab package insert was arthralgia. It is unknown why arthralgia was the most common $\mathrm{AE}$ in this analysis. However, rates of arthralgia in the brodalumab AMAGINE-2/-3 clinical trials (4.6\% and $5.8 \%$, respectively) were similar to those 
seen in clinical trials of the IL-17 antagonists secukinumab through 52 weeks $(8.1 \%)$ and ixekizumab through 24 weeks $(4.4 \%)[2,10,11]$. Additionally, the rate of arthralgia in the 1-year report was similar to that presented here $(0.07$ and 0.04 per PY, respectively) [7]. Finally, in a long-term analysis of the AMAGINE-1 trial, rates of arthralgia improved from $11.6 \%$ in year 1 to $4.1 \%$ in year 3 [5].

This pharmacovigilance report adds to a variety of recent safety meta-analyses for brodalumab and other biologic therapies for psoriasis. For instance, in a 2019 analysis, brodalumab was ranked as favorable regarding treatment discontinuation, but a lack of infection data was noted due to incomplete data [12]. Another meta-analysis that included brodalumab as well as other IL-17, IL-12/23, and IL23 antagonists reported that brodalumab $210 \mathrm{mg}$ ranked fourth out of 13 dosage options for short-term risk of AEs and eleventh out of 13 dosage options for short-term risk of serious AEs [13]. An analysis of brodalumab relative to placebo showed a small increase in overall AEs in the brodalumab group, with no significant differences between groups for serious AEs, nasopharyngitis, or upper respiratory tract infections [14]. The current report adds to these findings by reporting real-world pharmacovigilance data through 2 years, including serious infection data.

A potential limitation of this analysis is that only AEs reported to Ortho Dermatologics were documented. Additionally, reported patientexposure estimates, which were derived from 13 specialty pharmacies and noncontracted pharmacies that filled brodalumab prescriptions using a copay reimbursement card, do not account for patients' time on medication when treated outside of these pharmacies.

\section{CONCLUSION}

Many factors must be considered when selecting the optimal therapy for patients with psoriasis [15-17]. The consistent safety profile of brodalumab reported in pivotal trials and through long-term data is supported by this pharmacovigilance report. Furthermore, the absence of suicides or suicide attempts is notable.

\section{ACKNOWLEDGEMENTS}

Funding. This study was sponsored by Ortho Dermatologics. The journal's Rapid Service Fee was also funded by Ortho Dermatologics. Ortho Dermatologics is a division of Bausch Health US, LLC.

Authorship. All named authors meet the International Committee of Medical Journal Editors (ICMJE) criteriafor authorship for this article, take responsibility for the integrity of the work as a whole, and have given their approval for this version to be published.

Medical Writing and Editorial Assistance. Medical writing and editorial assistance were provided under the direction of the authors by Sara Gibson, PhD, Deirdre Rodeberg, $\mathrm{PhD}$, and Jenna Lewis, MA, ELS, of MedThink SciCom. Support for this assistance was funded by Ortho Dermatologics, a division of Bausch Health US, LLC.

Prior Presentation. Data presented in this poster have been previously presented in part at the Fall Clinical Dermatology Conference ${ }^{\circledR}$; October 29-November 1, 2020; Virtual and the 21st Annual Las Vegas Dermatology Seminar ${ }^{\circledR}$; November 20-21, 2020; Virtual.

Disclosures. Mark Lebwohl is an employee of Mount Sinai and receives research funds from AbbVie, Amgen, Arcutis Biotherapeutics, Boehringer Ingelheim, Dermavant, Eli Lilly, Incyte, Janssen Research \& Development, LLC, LEO Pharma, Ortho Dermatologics (a division of Bausch Health US, LLC), Pfizer, and UCB, Inc.; he is a consultant for Aditum Bio, Allergan, Almirall, Arcutis Biotherapeutics, Avotres Therapeutics, BirchBioMed Inc., BMD Skincare, Boehringer Ingelheim, Bristol-Myers Squibb, Cara Therapeutics, Castle Biosciences, Corrona, Dermavant Sciences, Evelo, Facilitate International Dermatologic Education, Foundation for 
Research and Education in Dermatology, Inozyme Pharma, Kyowa Kirin, LEO Pharma, Meiji Seika Pharma, Menlo, Mitsubishi, Neuroderm, Pfizer, Promius/Dr. Reddy's Laboratories, Serono, Theravance, and Verrica. Craig Leonardi is or has been a consultant, investigator, or speaker for AbbVie, Actavis, Allergan, Amgen, Boehringer Ingelheim, Celgene, Cellceutix, Coherus, Corrona, Dermira, Eli Lilly, Galderma, Glenmark, Janssen, LEO Pharma, Merck, Novartis, Novella, Pfizer, Sandoz, Sienna, Stiefel, Sun Pharmaceutical, UCB, Vitae, and Wyeth. Jashin $\mathrm{J}$. Wu is or has been an investigator, consultant, or speaker for AbbVie, Almirall, Amgen, Arcutis, Boehringer Ingelheim, BristolMyers Squibb, Dermavant, Dr. Reddy's Laboratories, Eli Lilly, Galderma, Janssen, LEO Pharma, Novartis, Regeneron, Sanofi Genzyme, Sun Pharmaceutical, UCB, and Valeant Pharmaceuticals North America LLC. April Armstrong is or has been a research investigator for and/or scientific advisor to AbbVie, BMS, Dermavant, Dermira, Incyte, Janssen, LEO Pharma, Lilly, Modmed, Novartis, Ortho Dermatologics (a division of Bausch Health US, LLC), Pfizer, Regeneron, Sanofi, Sun Pharmaceutical, and UCB. Nicole Rawnsley and Abby Jacobson are employees of Ortho Dermatologics (a division of Bausch Health US, LLC). Mohammed Merchant was an employee of Bausch Health during the study conduction and manuscript development; he is currently affiliated with Organon \& Co., a wholly owned subsidiary of Merck \& Co., Inc., Kenilworth, NJ, USA. Binu Alexander is an employee of Bausch Health.

Compliance with Ethics Guidelines. This article is based on previously conducted studies and does not contain any new studies with human participants or animals performed by any of the authors.

Data Availability. The datasets generated or analyzed during the current study are available from the corresponding author on reasonable request.

Open Access. This article is licensed under a Creative Commons Attribution-NonCommercial 4.0 International License, which permits any non-commercial use, sharing, adaptation, distribution and reproduction in any medium or format, as long as you give appropriate credit to the original author(s) and the source, provide a link to the Creative Commons licence, and indicate if changes were made. The images or other third party material in this article are included in the article's Creative Commons licence, unless indicated otherwise in a credit line to the material. If material is not included in the article's Creative Commons licence and your intended use is not permitted by statutory regulation or exceeds the permitted use, you will need to obtain permission directly from the copyright holder. To view a copy of this licence, visit http://creativecommons.org/licenses/by$\mathrm{nc} / 4.0 /$.

\section{REFERENCES}

1. Siliq [package insert]. Bridgewater, NJ: Bausch Health US, LLC. 2017.https://www.accessdata.fda. gov/drugsatfda_docs/label. Accessed 14 Dec 2020.

2. Lebwohl M, Strober B, Menter A, et al. Phase 3 studies comparing brodalumab with ustekinumab in psoriasis. N Engl J Med. 2015;373(14):1318-28.

3. Papp KA, Reich K, Paul C, et al. A prospective phase III, randomized, double-blind, placebo-controlled study of brodalumab in patients with moderate-tosevere plaque psoriasis. Br J Dermatol. 2016;175(2): 273-86.

4. Papp KA, Leonardi C, Menter A, et al. Brodalumab, an anti-interleukin-17-receptor antibody for psoriasis. N Engl J Med. 2012;366(13):1181-9.

5. Papp K, Menter A, Leonardi C, et al. Long-term efficacy and safety of brodalumab in psoriasis through 120 weeks and after withdrawal and retreatment: subgroup analysis of a randomized phase III trial (AMAGINE-1). Br J Dermatol. 2020. https://doi.org/10.1111/bjd.19132.

6. Puig L, Lebwohl M, Bachelez H, Sobell J, Jacobson AA. Long-term efficacy and safety of brodalumab in the treatment of psoriasis: 120-week results from the randomized, double-blind, placebo- and active comparator-controlled phase 3 AMAGINE-2 trial. J Am Acad Dermatol. 2020;82(2):352-9.

7. Lebwohl M, Leonardi $\mathrm{C}, \mathrm{Wu} \mathrm{JJ}$, et al. One-year pharmacovigilance update of brodalumab [editorial]. J Drugs Dermatol. 2020;19(8):807-8. 
8. Centers for Disease Control and Prevention, National Center for Immunization and Respiratory Diseases. The flu season [Internet]. Available from: https://www.cdc.gov/flu/about/season/flu-season. htm. 2018. Accessed 19 Oct 2020.

9. Gottlieb A, Lebwohl M, Liu C, Israel RJ, Jacobson A. Malignancy rates in brodalumab clinical studies for psoriasis. Am J Clin Dermatol. 2020;21(3):421-30.

10. Blauvelt A, Reich K, Tsai TF, et al. Secukinumab is superior to ustekinumab in clearing skin of subjects with moderate-to-severe plaque psoriasis up to 1 year: results from the CLEAR study. J Am Acad Dermatol. 2017;76(1):60-9.

11. Reich K, Pinter A, Lacour JP, et al. Comparison of ixekizumab with ustekinumab in moderate-to-severe psoriasis: 24 -week results from IXORA-S, a phase III study. Br J Dermatol. 2017;177(4):1014-23.

12. Xu G, Xia M, Jiang C, et al. Comparative efficacy and safety of thirteen biologic therapies for patients with moderate or severe psoriasis: a network metaanalysis. J Pharmacol Sci. 2019;139(4):289-303.

13. Bai F, Li GG, Liu Q, Niu X, Li R, Ma H. Short-term efficacy and safety of IL-17, IL-12/23, and IL-23 inhibitors brodalumab, secukinumab, ixekizumab, ustekinumab, guselkumab, tildrakizumab, and risankizumab for the treatment of moderate to severe plaque psoriasis: a systematic review and network meta-analysis of randomized controlled trials. J Immunol Res. 2019;2019:2546161.

14. Attia A, Abushouk AI, Ahmed H, et al. Safety and efficacy of brodalumab for moderate-to-severe plaque psoriasis: a systematic review and meta-analysis. Clin Drug Investig. 2017;37(5):439-51.

15. Amin M, No DJ, Egeberg A, Wu JJ. Choosing firstline biologic treatment for moderate-to-severe psoriasis: what does the evidence say? Am J Clin Dermatol. 2018;19(1):1-13.

16. Kaushik SB, Lebwohl MG. Psoriasis: which therapy for which patient: focus on special populations and chronic infections. J Am Acad Dermatol. 2019;80(1):43-53.

17. Kaushik SB, Lebwohl MG. Psoriasis: which therapy for which patient: psoriasis comorbidities and preferred systemic agents. J Am Acad Dermatol. 2019;80(1):27-40. 